\title{
The efficient low-mass Seyfert MCG-05-23-016 ^
}

\author{
V. Beckmann ${ }^{1,2,3}$, T. J. -L. Courvoisier ${ }^{1,2}$, N. Gehrels ${ }^{4}$, P. Lubiński $1^{1,5}$, J. Malzac ${ }^{6}$, P.-O. Petrucci ${ }^{7}$, \\ C. R. Shrader ${ }^{4}$, and S. Soldi ${ }^{1,2}$
}

1 ISDC Data Centre for Astrophysics, Chemin d'Écogia 16, 1290 Versoix, Switzerland e-mail: Volker.Beckmann@unige.ch

2 Observatoire Astronomique de l'Université de Genève, Chemin des Maillettes 51, 1290 Sauverny, Switzerland

3 University of Maryland Baltimore County, 1000 Hilltop Circle, Baltimore, MD 21250, USA

${ }^{4}$ Astrophysics Science Division, NASA Goddard Space Flight Center, Code 661, MD 20771, USA

5 Centrum Astronomiczne im. M. Kopernika, Bartycka 18, PL-00-716 Warszawa, Poland

${ }^{6}$ Centre d'Étude Spatiale des Rayonnements (CESR), OMP, UPS, CNRS, BP 44346, 31028 Toulouse Cedex 4, France

7 Laboratoire d'Astrophysique de Grenoble, Univ. J. Fourier/CNRS, UMR 5571, BP 53X, 38041 Grenoble Cedex, France

Received 25 July 2008 / Accepted 10 October 2008

\section{ABSTRACT}

Aims. The Seyfert 1.9 galaxy MCG-05-23-016 has been shown to exhibit a complex X-ray spectrum. This source has moderate $\mathrm{X}$-ray luminosity, hosts a comparably low-mass black hole, but accretes at a high Eddington rate, and allows us to study a super massive black hole in an early stage.

Methods. Three observations of the INTEGRAL satellite simultaneous with pointed Swift/XRT observations performed from December 2006 to June 2007 are used in combination with public data from the INTEGRAL archive to study the variability of the hard X-ray components and to generate a high-quality spectrum from 1 to $150 \mathrm{keV}$.

Results. The AGN shows little variability in the hard X-ray spectrum, with some indication of a variation in the high-energy cut-off energy ranging from $50 \mathrm{keV}$ to $\gg 100 \mathrm{keV}$, with an electron plasma temperature in the $10-90 \mathrm{keV}$ range. The reflection component is not evident and, if present, the reflected fraction can be constrained to $R<0.3$ for the combined data set. Comparison to previous observations shows that the reflection component has to be variable. No variability in the UV and optical range is observed on a time scale of 1.5 years.

Conclusions. The hard X-ray spectrum of MCG-05-23-016 appears to be stable with the luminosity and underlying power law varying moderately and the optical/UV flux staying constant. The reflection component and the iron K $\alpha$ line seem to have decreased between December 2005 and the observations presented here. The spectral energy distribution appears to be similar to that of Galactic black hole systems, e.g. XTE $1118+480$ in the low state. The AGN exhibits a remarkably high Eddington ratio of $L_{\mathrm{bol}} / L_{\mathrm{Edd}} \gtrsim 0.8$ (or $L_{\mathrm{bol}} / L_{\mathrm{Edd}} \gtrsim 0.1$, if we consider a higher mass of the central engine) and, at the same time, a low cut-off energy around $70 \mathrm{keV}$. Objects like MCG-05-23-016 might indicate the early stages of super massive black holes, in which a strong accretion flow feeds the central engine.

Key words. galaxies: active - galaxies: Seyfert - X-rays: galaxies - galaxies: individual: MCG-05-23-016 -

accretion, accretion discs

\section{Introduction}

Active galactic nuclei (AGN) are commonly assumed to be super massive black holes in the centre of galaxies, in which accretion processes give rise to emission throughout the electromagnetic spectrum. AGN are observed to date up to redshifts of $z \sim 6.4$ (Willott et al. 2007), showing that super massive black holes with masses of $M_{\mathrm{BH}} \sim 10^{8} M_{\odot}$ must have been formed as early as $<0.7$ Gyrs after the formation of the first stars (Kashlinsky et al. 2005). In order to be able to form super massive black holes at this early stage of the Universe, merging events and high accretion rates are required. This black hole evolution is closely tied to the growth of the bulge of the AGN's host galaxy, as both seem to be correlated with $M_{\mathrm{BH}} \simeq 10^{-3} M_{\mathrm{Bulge}}$ (Gebhardt et al. 2000).

* Based on observations with INTEGRAL, an ESA project with instruments and science data centre funded by ESA member states (especially the PI countries: Denmark, France, Germany, Italy, Switzerland, Spain), Czech Republic and Poland, and with the participation of Russia and the USA.
Recent studies intend to not only find unification models for the different AGN types but also probe whether their central engines and super massive black holes are simply up-scaled versions of Galactic black holes like Cyg X-1, GRO J1655-40, or GX 339-4. In this context, models of black hole accretion can be tested using objects showing extreme physical properties, like luminosity, accretion rate, or Eddington ratio. The Seyfert 1.9 galaxy presented here resides in an extreme end of the known parameter space of AGN. X-ray observations give a direct view on matter close to the super massive black hole, providing insights into the geometry and the state of the matter. The flux and spectral variability of the sources in the hard X-rays reflect the size and physical state of the regions involved in the emission processes (see Uttley \& McHardy 2004, for a brief review).

MCG-05-23-016 is one of the brightest Seyfert galaxies in the X-rays. This Seyfert 1.9 galaxy at redshift $z=$ 0.0085 has not only been studied in the $2-10 \mathrm{keV}$ band by most X-ray missions so far, but has also been detected in hard X-rays above $20 \mathrm{keV}$ with BeppoSAX/PDS (e.g. Balestra et al. 2004), INTEGRAL/IBIS (Soldi et al. 2005), and Swift/BAT 
Table 1. Observation log of INTEGRAL and Swift/XRT observations on MCG-05-23-016.

\begin{tabular}{ccrrrr}
\hline \hline $\begin{array}{c}\text { INTEGRAL } \\
\text { revolution }\end{array}$ & Time covered & ISGRI exposure & JEM-X exp. & Swift/XRT & Exposure \\
{$[$ U.T. } & {$[\mathrm{ks}]$} & 69.3 & - & $05-12-09$ & 10.3 \\
\hline $256-282$ & $04-11-18-05-02-05$ & 26.3 & 21.7 & $06-12-13$ & 1.9 \\
508 & $06-12-12-06-12-13$ & 128.7 & 122.0 & $07-05-31$ & 2.1 \\
565 & $07-05-30-07-06-01$ & 89.4 & 79.8 & $07-06-13$ & 2.9 \\
569 & $07-06-12-07-06-13$ & & &
\end{tabular}

(Beckmann et al. 2007; Tueller et al. 2008). With a bolometric luminosity of $L_{\mathrm{bol}} \simeq 2 \times 10^{44} \mathrm{erg} \mathrm{s}^{-1}$ this Seyfert is a moderately luminous object, with a comparably small central black hole of $M_{\mathrm{BH}}=2 \times 10^{6} M_{\odot}$ (Wang \& Zhang 2007). Wang \& Zhang used the width of the OIII line (Greene \& Ho 2005) to obtain the black hole mass from the $M_{\mathrm{BH}}-\sigma$ relation (Tremaine et al. 2002) and give the error of the measurement with 0.7 dex.

Balestra et al. (2004) derived from ASCA, BeppoSAX, Chandra and XMM-Newton data that the iron $\mathrm{K} \alpha$ line apparent in the spectrum seems to be a superposition of a narrow and a broad component, that a reflection component of $R=0.45$ is detectable, and that the continuum can be modeled by a photon index of $\Gamma \simeq 1.7$ and a cut-off at $E_{\mathrm{C}}=110 \mathrm{keV}$. The observations covered a time span from 1994 to 2001, during which the object showed little flux variation and a stable spectrum as well as constant iron line and reflection component. A recent re-analysis of the BeppoSAX data by Dadina (2007) indicates a slightly steeper spectrum $\left(\Gamma=1.79_{-0.08}^{+0.07}\right)$ with higher cutoff energy $\left(E_{\mathrm{c}}=191_{-60}^{+110} \mathrm{keV}\right)$, and stronger reflection component $\left(R=0.74_{-0.52}^{+0.22}\right)$. An analysis of $R X T E$ data by Mattson $\&$ Weaver (2004) using a fixed underlying power law slope of $\Gamma=1.88$ led to a variable reflection component in the range $R=0.35-0.57$ and iron $K \alpha$ line flux $\left(f_{K \alpha}=(1.55-1.99) \times\right.$ $10^{-4}$ photons $\left.\mathrm{keV}^{-1} \mathrm{~cm}^{-2} \mathrm{~s}^{-1}\right)$. Recently, observations on MCG05-23-016 by Suzaku showed a 0.4-100 keV spectrum which appeared to be a cut-off power law of $\Gamma=1.9$ and $E_{\mathrm{C}}>170 \mathrm{keV}$ plus a dual reflector with $R \sim 0.9$ and $R \sim 0.5$, respectively (Reeves et al. 2007). XMM-Newton and Chandra data confirmed that the iron $\mathrm{K} \alpha$ line complex consists of a broad and of a narrow component and revealed evidence for outflowing material (Braito et al. 2007). The exact shape and structure of the highenergy spectrum of MCG-05-23-016 remains elusive though. INTEGRAL and Swift observations can help to shed light on the existence and position of the high-energy cut-off and variability of the hard X-ray spectrum. In Sect. 2 we describe the analysis of the INTEGRAL and simultaneous Swift observations. We discuss the findings in Sect. 3, with emphasis on similarities with Galactic black hole systems and on the fact that the Seyfert galaxy apparently operates at high Eddington ratio. Conclusions are presented in Sect. 4.

\section{Observations and data analysis}

The INTEGRAL (Winkler et al. 2003) mission offers the unique possibility to perform simultaneous observations of AGN over the 3-1000 keV energy region. This is achieved by the X-ray monitor (3-35 $\mathrm{keV}$ ) JEM-X (Lund et al. 2003), the soft gamma-ray imager (18-1000 keV) IBIS/ISGRI (Lebrun et al. 2003), and the spectrograph SPI (Vedrenne et al. 2003), which operates in the 20-8000 keV region. Each of these instruments employs the coded-aperture technique.
INTEGRAL performed three dedicated pointed observations on MCG-05-23-016 in December 2006 (spacecraft revolution 508) and in May/June 2007 (revolution 565 and 569). As observing strategy hexagonal dithering has been chosen in order to achieve a sufficient coverage also by the JEM-X monitor. During these observations the spectrometer SPI was not available because it went through an annealing cycle. In addition and for comparison reasons, we analysed INTEGRAL data on MCG-0523-016 which had been taken prior to our observations. During all these measurements the source was too far off-axis to be covered by JEM-X. Reliable data from the SPI spectrometer can be taken also at larger off-axis angles. Therefore the public SPI data include more revolutions than the IBIS/ISGRI data analysed here. All INTEGRAL data were analysed using the Offline Science Analysis (OSA) software version 7.0 distributed by the ISDC (Courvoisier et al. 2003). For IBIS/ISGRI standard spectral extraction was applied, using 12 spectral bins in the $18 \mathrm{keV}$ to $521 \mathrm{keV}$ energy range. For JEM-X the flux values were extracted from imaging analysis in four energy bands (3-6-10$15-35 \mathrm{keV})$, which provides more reliable results for a source at the flux level of MCG-05-23-016 than standard spectral extraction.

In order to achieve a wider spectral energy coverage, pointed Swift observations were performed simultaneous with the INTEGRAL ones. Swift (Gehrels et al. 2004) provides two narrow-field instruments, the XRT (Burrows et al. 2005) with energy range $0.3-10.0 \mathrm{keV}$ and the UVOT (Roming et al. 2005) which is equipped with 6 filters in the optical and UV range. The XRT and UVOT data have been analysed using HEADAS version 6.1.1 applying latest calibration files as available in January 2008.

The INTEGRAL IBIS/ISGRI and JEM-X1, as well as the Swift pointed observations are summarised in Table 1 . The observations in INTEGRAL revolution 508, 565, and 569 are truly simultaneous with Swift pointed observations, while the public data obtained from revolution 256 to 282 are separated from the Swift observation by at least 10 months and also do not include any JEM-X data and therefore no information about the 9-18 keV energy range. The SPI data showed only a marginal detection on the $4 \sigma$ level in the $20-40 \mathrm{keV}$ energy band in the combined data set. The SPI spectrum can be fit by a single power law model with photon index $\Gamma=1.8_{-0.6}^{+1.51}$. As these data are not strictly simultaneous with the other observations and are of low significance, the SPI data have not been considered in the following analysis of MCG-05-23-016.

The data of IBIS/ISGRI, JEM-X, and Swift/XRT have been fit simultaneously using XSPEC version 11.3.2 (Arnaud 1996). As the most simplistic approach an absorbed power law model has been fit to the data. Only in the case of revolution 508 data this model gives an acceptable fit with $\chi_{v}^{2}=1.06$ for 55 degrees of freedom. In the other cases, a good fit was achieved

\footnotetext{
${ }^{1}$ Errors are $2 \sigma$ errors throughout the paper.
} 
Table 2. Spectral fitting of combined Swift/XRT and INTEGRAL ISGRI/JEM-X1 data of MCG-05-23-016 using an absorbed cut-off power law model.

\begin{tabular}{cccccc}
\hline \hline $\begin{array}{c}\text { INTEGRAL } \\
\text { revolution }\end{array}$ & $\begin{array}{c}N_{\mathrm{H}} \\
{\left[10^{22} \mathrm{~cm}^{-2}\right.}\end{array}$ & $\Gamma$ & $\begin{array}{c}E_{\mathrm{C}} \\
{[\mathrm{keV}]}\end{array}$ & $\chi_{v}^{2}$ & d.o.f. \\
\hline $256-282$ & $1.26_{-0.07}^{+0.07}$ & $1.49_{-0.08}^{+0.08}$ & $57_{-16}^{+31}$ & 1.05 & 276 \\
508 & $1.29_{-0.14}^{+0.15}$ & $1.76_{-0.08}^{+0.08}$ & $>90$ & 1.06 & 55 \\
565 & $1.24_{-0.14}^{+0.13}$ & $1.51_{-0.10}^{+0.09}$ & $86_{-29}^{+68}$ & 0.95 & 80 \\
569 & $1.04_{-0.10}^{+0.09}$ & $1.42_{-0.09}^{+0.09}$ & $65_{-18}^{+35}$ & 0.92 & 97 \\
all data & $1.24_{-0.05}^{+0.05}$ & $1.52_{-0.05}^{+0.07}$ & $72_{-14}^{+21}$ & 0.99 & 268 \\
\hline
\end{tabular}

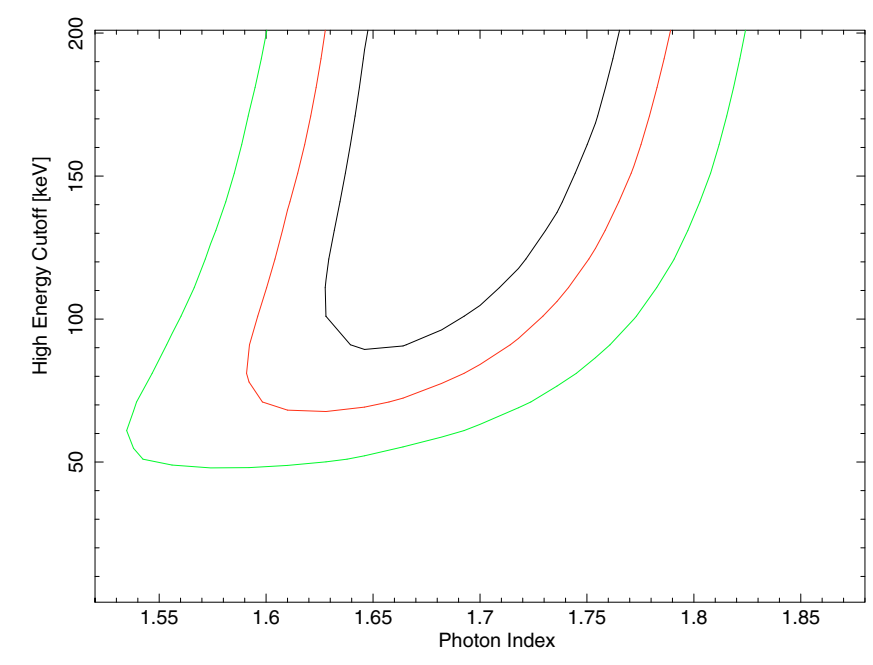

Fig. 1. Contour plot for an absorbed cut-off power law applied to the combined Swift/XRT, INTEGRAL JEM-X1, and IBIS/ISGRI data obtained in December 2006. The contours show the allowed range for the photon index and the cut-off energy at the 1,2 and 3 sigma level. No cut-off is required by the data.

with an absorbed cut-off power law model. The fit results are summarised in Table 2.

All spectra require a high-energy cut-off in the range $60-85 \mathrm{keV}$. The only exception is the short exposure in revolution 508. In this case, the existence of a cut-off at $50 \mathrm{keV}, 70 \mathrm{keV}$ and $90 \mathrm{keV}$, can be rejected at a significance level of $3 \sigma, 2 \sigma$, and $1 \sigma$, respectively, as shown in Fig. 1.

None of the spectra required an additional reflection component. Applying a Compton reflection model, such as pexrav (Magdziarz \& Zdziarski 1995) which describes a reflection component from cold material using a cut-off power-law as continuum, shows that the existence of such a component in MCG-05-23-016 can neither be proven nor rejected by the single spectra. The soft X-ray excess below $0.8 \mathrm{keV}$, as reported in Reeves et al. (2007), is apparent only in Swift/XRT spectrum of December 2005.

As the spectral parameters listed in Table 2 appear to be nearly constant, we also applied a fit to the total combined data set as shown in Fig. 2. The total combined spectrum can be fit by an absorbed cut-off power law model with $N_{\mathrm{H}}=$ $(1.24 \pm 0.05) \times 10^{22} \mathrm{~cm}^{-2}$, photon index $\Gamma=(1.52 \pm 0.05)$, cutoff energy $E_{\mathrm{c}}=72_{-14}^{+21} \mathrm{keV}$. The fit results in $\chi_{268}^{2}=0.99$. The significance of the high energy cut-off and the tight constraint on the spectral slope can be seen in the contour plot shown in Fig. 3. For this combined spectrum also the strength of a possible Compton reflection component can be constrained. Applying the pexrav model and using the same relative iron abundance

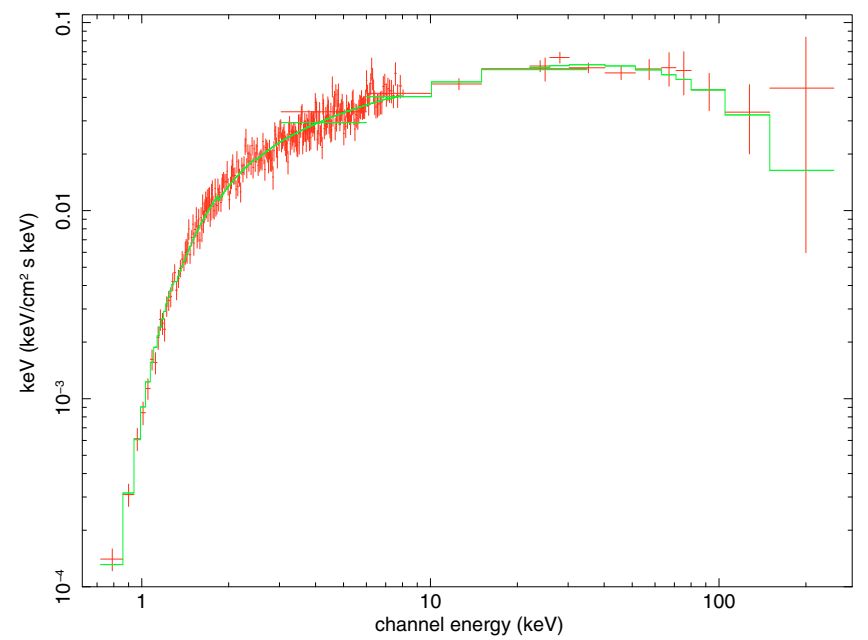

Fig. 2. Combined spectrum of all Swift/XRT, INTEGRAL JEM-X1, and IBIS/ISGRI data obtained between November 2004 and June 2007. The absorbed cut-off power law fit results in $N_{\mathrm{H}}=(1.24 \pm 0.05) \times 10^{22} \mathrm{~cm}^{-2}$, photon index $\Gamma=1.52 \pm 0.05$, and cut-off energy $E_{\mathrm{c}}=72_{-14}^{+21} \mathrm{keV}$ for a $\chi_{268}^{2}=0.99$.

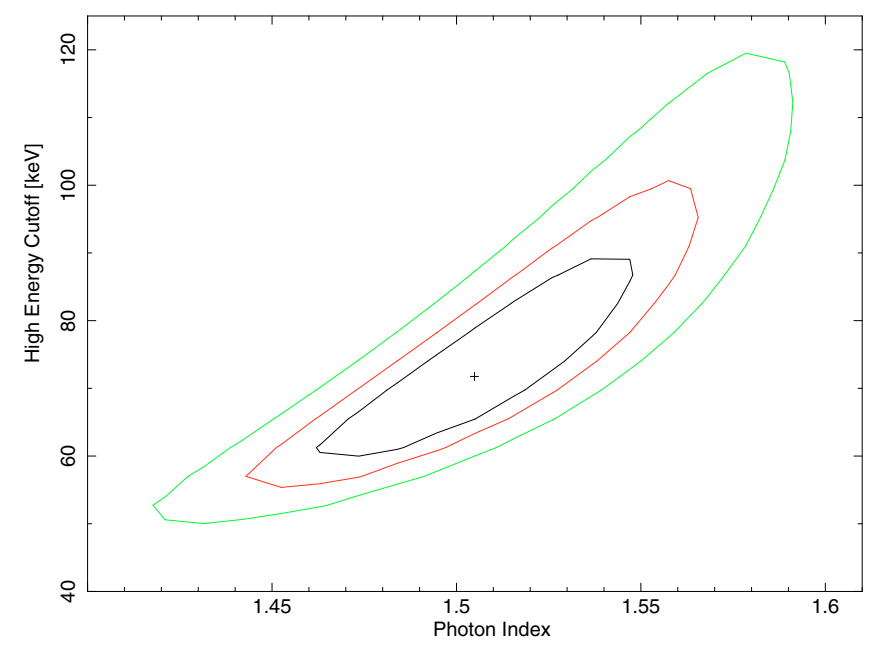

Fig. 3. Contour plot for an absorbed cut-off power law applied to all the combined Swift/XRT, INTEGRAL JEM-X1, and IBIS/ISGRI data listed in Table 1. The contours show the allowed range for the photon index and the cut-off energy at the 1,2 and 3 sigma level.

of 0.4 and inclination angle of $45^{\circ}$ as Reeves et al. (2007) result in similar absorption, photon index and cut-off energy $\left(N_{\mathrm{H}}=\right.$ $\left.1.25_{-0.05}^{+0.06} \times 10^{22} \mathrm{~cm}^{-2}, \Gamma=1.52_{-0.05}^{+0.07}, E_{\mathrm{f}}=72_{-14}^{+21} \mathrm{keV}\right)$, and gives a relative reflection strength of $R=0.06_{-0.06}^{+0.20}\left(\chi_{267}^{2}=0.99\right)$. The compPS model developed by Poutanen \& Svensson (1996) for a plane-parallel slab geometry using exact numerical solution of the radiative transfer equation indicates a stronger reflection component $\left(R=0.4_{-0.2}^{+0.3}\right.$ with an electron temperature of $k T_{\mathrm{e}}=113_{-10}^{+15} \mathrm{keV}\left(\chi_{265}^{2}=1.04\right)$. It has to be pointed out though that this model is a significantly worse representation of the data than the pexrav or the simple cut-off power law model. Thus, also in the overall combined spectrum a reflection component is not required, and can be constrained to $R<0.25$ on a $2 \sigma$ level. Adding a Gaussian line at the position of the $\mathrm{FeK}_{\alpha}$ line gives an equivalent width of $E W=61_{-40}^{+47} \mathrm{eV}$, although this component is not statistical significant.

We then left the iron abundance as a free parameter in the pexrav model and forced the iron line flux to have an equivalent 
width of $100 \mathrm{eV}$ with respect to the reflection continuum alone. Applying this model, one finds that the iron abundance is not constrained by the data and one gets similar results with a marginal evidence for a reflection component of $R=0.13_{-0.13}^{+0.18}$ consistent with no reflection, a cut-off energy of $E_{\mathrm{f}}=77_{-18}^{+17} \mathrm{keV}$, and photon index $\Gamma=1.56_{-0.09}^{+0.05}\left(\chi_{265}^{2}=1.02\right)$.

The Swift pointed observations also provide flux information about the $U V$ and the optical $V, B$, and $U$ bands. All four Swift/UVOT measurements revealed the same fluxes within the error of $0.1 \mathrm{mag}$. The results were: $V=13.9 \mathrm{mag}, B=14.9 \mathrm{mag}$, $U=15.3 \mathrm{mag}, U V W 1=16.1 \mathrm{mag}, U V M 2=17.0 \mathrm{mag}$, and $U V W 2=16.9 \mathrm{mag}$.

\subsection{Detection of $1 R X S$ J092418.0-314212}

Within the JEM-X data set covering the 3-35 keV energy range, another source is clearly detected in the field of view during the observation of MCG-05-23-016. The source position coincides with 1RXS J092418.0-314212, currently an unidentified object. The source is not detectable in the IBIS/ISGRI data set and the source position is not within the Swift/XRT field of view. The JEM-X data cannot be represented by a power law model, but are well represented by a blackbody model with temperature $k T=1.4_{-0.2}^{+0.3} \mathrm{keV}$. We therefore suggest that this source is likely to be a Galactic X-ray binary system and that the spectrum shows the black body radiation emitted from the central parts of its accretion disc.

\section{Discussion}

\subsection{The hard $X$-ray emission}

In our study of MCG-05-23-016 we have made use of the hard $\mathrm{X}$-ray capabilities of INTEGRAL, and in particular of the imager IBIS/ISGRI, in combination with the soft X-ray spectrum provided by Swift/XRT. Although the combined spectrum clearly does not provide the wealth of information in the soft X-rays as shown in XMM-Newton, Chandra, and Suzaku data, the better sensitivity at energies above $30 \mathrm{keV}$ puts significant constraints on the hard X-ray component. First of all, a high-energy cutoff in the range $57 \mathrm{keV}$ to $86 \mathrm{keV}$ is required in most of the observed spectra. However, one observation in December 2006 shows a spectrum extending up to $\sim 200 \mathrm{keV}$, excluding a cut-off at $50 \mathrm{keV}$ on a $3 \sigma$ level, and at $90 \mathrm{keV}$ on a $1 \sigma$ level, even when allowing for a different photon index of the underlying power law at the same time (Fig. 1). It is important to note though, that the spectrum in this case is significantly steeper $(\Gamma=1.7 \pm 0.1)$ than in the cases where a cut-off is measured $(\Gamma=1.5 \pm 0.1)$.

The differences in the hard X-ray spectrum, i.e. the differences in high-energy cut-off and spectral slope, can be interpreted as different temperatures of the electron plasma, which is the source of the inverse Compton emission thought to be the dominant component in this energy range. A physical model describing Comptonization of soft photons by a hot plasma, the so called compTT model, has been developed by (Titarchuk 1994). The model includes the plasma temperature $T_{\mathrm{e}}$ of the hot corona, the optical depth $\tau_{\mathrm{p}}$ of this plasma, and the temperature $T_{0}$ of the soft photon spectrum. Because the spectrum starts at $E \simeq$ $0.7 \mathrm{keV}, T_{0}$ is not well constrained by the data and has been fixed to $10 \mathrm{eV}$. Table 3 gives the results of this model for the individual data sets as well as for the combined spectrum for plane geometry. As for the cut-off energy $E_{\mathrm{C}}$, the variation of the plasma temperature is significant on a $2 \sigma$ level. The highest plasma
Table 3. Thermal Comptonisation model (compTT) applied to combined MCG-05-23-016 data.

\begin{tabular}{ccccccc}
\hline \hline $\begin{array}{c}\text { INTEGRAL } \\
\text { revolution }\end{array}$ & $\begin{array}{c}N_{\mathrm{H}} \\
{\left[10^{22} \mathrm{~cm}^{-2}\right]}\end{array}$ & $\begin{array}{c}k T_{\mathrm{e}} \\
{[\mathrm{keV}]}\end{array}$ & $\tau_{\mathrm{p}}$ & $\begin{array}{c}L_{2-100 \mathrm{keV}} \\
{\left[\mathrm{erg} \mathrm{s}^{-1}\right]}\end{array}$ & $\chi_{v}^{2}$ & d.o.f. \\
\hline $256-282$ & $1.33_{-0.07}^{+0.07}$ & $11_{-3}^{+15}$ & $3.4_{-1.0}^{+0.8}$ & $4.3 \times 10^{43}$ & 1.08 & 275 \\
508 & $1.21_{-0.11}^{+0.13}$ & $90_{-73}^{+76}$ & $0.5_{-0.4}^{+2.4}$ & $4.6 \times 10^{43}$ & 0.97 & 49 \\
565 & $1.35_{-0.11}^{+0.11}$ & $23_{-7}^{+105}$ & $2.1_{-1.8}^{+0.6}$ & $4.2 \times 10^{43}$ & 0.91 & 79 \\
569 & $1.14_{-0.08}^{+0.08}$ & $14_{-3}^{+5}$ & $3.1_{-0.5}^{+0.6}$ & $4.4 \times 10^{43}$ & 0.92 & 96 \\
all data & $1.33_{-0.04}^{+0.04}$ & $18_{-3}^{+7}$ & $2.5_{-0.6}^{+0.4}$ & $4.4 \times 10^{43}$ & 1.04 & 268 \\
\hline
\end{tabular}

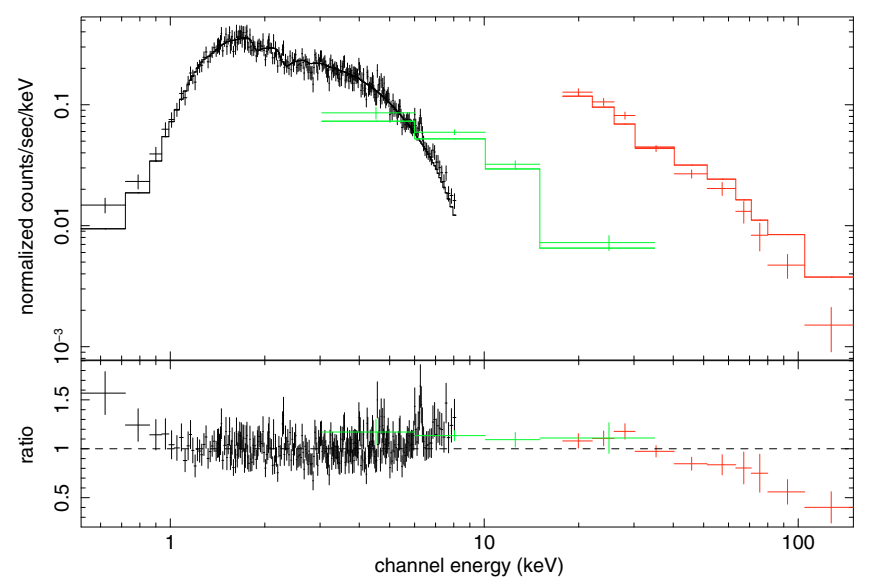

Fig. 4. Absorbed single power law model fit applied to the $2-5 \mathrm{keV}$ range of the combined data set. The ratio between the data and the model shows the soft excess at energies below $1 \mathrm{keV}$, the iron $\mathrm{K} \alpha$ line, the significant cut-off at high energies, but no trace of a reflection component.

temperature and lowest optical depth is observed during revolution 508, when the cut-off in the hard X-ray spectrum disappeared (Table 2). However, it has to be taken into account that the rev. 508 spectrum can also be fit by applying the same compTT model which gives the best fit to all data (i.e. with $k T_{\mathrm{e}}=18 \mathrm{keV}$ and $\left.\tau_{\mathrm{p}}=2.5\right)$ with an acceptable fit result $\left(\chi_{v}^{2}=1.09\right.$ for 52 d.o.f.).

Similar values for cut-off energy and photon index as presented here were derived previously based on combined BeppoSAX and INTEGRAL IBIS/ISGRI data, resulting in $E_{\mathrm{C}}=$ $112_{-43}^{+40} \mathrm{keV}, \Gamma=1.74_{-0.14}^{+0.08}$, plus a reflection component with $R=1.2_{-1.0}^{+0.6}$ (Molina et al. 2006). As these observations are not simultaneous, these values have to be taken with some caution though, and the reflection component is not well constrained.

Apart from the variation of the cut-off energy and of the plasma temperature, describing a variable inverse Compton process, the low level of the reflection component in the data studied here is remarkable. Figure 4 shows a simple model fit applied to the combined data set, using an absorbed power law model fitted in the $2-5 \mathrm{keV}$ range as done by Reeves et al. (2007) to show the significance of the reflection component. The ratio between the data and the model shows several components: the soft excess at energies below $1 \mathrm{keV}$, the iron $\mathrm{K} \alpha$ line, and the significant cut-off at high energies. There is no evidence however of a significant reflection component.

The $2 \sigma$ upper limit is $R<0.25$, while in the observations presented by Reeves et al. (2007), a value of $R<0.7$ can be excluded on a $3 \sigma$ level. We have to conclude that the strong reflection component, visible in the December 2005 data taken by 
Suzaku, XMM-Newton and Chandra disappeared within a year. Also the excess observed at soft X-rays by Reeves et al. is only detectable in the Swift/XRT data from December 2005 and not afterwards. At the same time, also the iron line complex has decreased in flux, from an equivalent width of $E W=130 \pm 17 \mathrm{eV}$ in December 2005 to about $E W=61 \pm 45 \mathrm{eV}$ in $2006 / 2007$. Unfortunately the Swift/XRT data do not allow us to disentangle the broad and narrow line component of the line complex, as reported in Balestra et al. (2004) and also in Reeves et al. (2007). If we assume that the narrow and the broad line components arise from two different absorber, with the broad line being emitted closer and the narrow line further away from the central engine as proposed by Reeves et al., then we might indeed observe the disappearance of the broad iron line $(E W \simeq$ $60 \mathrm{eV}$; Reeves et al. 2007) and of the reflection component connected to the close absorber between the December 2005 and December 2006 observations, leaving the spectrum only with the narrow line component and reflection from the distant absorber. This disappearance is not accompanied by a significant change in X-ray luminosity or in UV/optical flux. The lack of hard X-ray flux variability appeared also in a study of MCG05-23-016 using Swift/BAT, which resulted in a marginal variability of $6 \pm 4 \%$ on a 20 day time scale (Beckmann et al. 2007). The soft X-ray spectrum in the range $2-10 \mathrm{keV}$ however appears to be variable by a factor of $\sim 1.7$ throughout historic observations. The Swift/XRT data presented here give a flux of $f_{2-10 \mathrm{keV}}=(7.1-8.1) \times 10^{-11} \mathrm{erg} \mathrm{cm}^{-2} \mathrm{~s}^{-1}$, with literature values in the range $f_{2-10 \mathrm{keV}}=(7.1-11.9) \times 10^{-11} \mathrm{erg} \mathrm{cm}^{-2} \mathrm{~s}^{-1}$ (e.g. Mattson \& Weaver 2004; Balestra et al. 2004).

Mattson \& Weaver (2004) also found variable Compton reflection when studying MCG-05-23-016 with RXTE and BeppoSAX. In their study, the strength of the reflection component is anti-correlated with the $2-10 \mathrm{keV}$ flux. In this context, one would expect a very high reflection component in the data presented here, where the $2-10 \mathrm{keV}$ flux is at the historic minimum. When using the same photon index of $\Gamma=1.77$ as Mattson \& Weaver, we get a relative reflection of $R=0.78_{-0.21}^{+0.26}$, but at the same time $\chi_{v}^{2}$ rises to 1.10 , compared to $\chi_{v}^{2}=0.99$ with no reflection and a photon index of $\Gamma=1.52$. We therefore do not consider the fixed spectral slope the valid approach to fit the data presented here. A similar behaviour, of a hardening of the spectra with decreasing reflection component as we see it from comparison with previous measurements, has been observed before. Zdziarski et al. (2004) analysed this relation for 61 Ginga observations of 24 radio quiet Seyfert galaxies. They found a strong correlation of the form $R=u \Gamma^{v}$ with the parameters $u=(1.4 \pm 1.2) \times 10^{-4}$ and $v=12.4 \pm 1.2$. The observed trend follows closely this relation. Our measurement of $\Gamma=1.5$ would result in $R=0.03 \pm 0.04$, consistent with the upper limit we derived. Also the results of Mattson \& Weaver and of Reeves et al. (2007) fit well on this correlation. The measurement of Molina et al. (2006) based on non-simultaneous BeppoSAX and INTEGRAL IBIS/ISGRI data however, with $R=1.2$ and $\Gamma=1.7$ lies about $7 \sigma$ off this relation which results in $R=0.13 \pm 0.16$. This might indicate that it is indeed essential to study simultaneous observations when determining the strength of the reflection component.

An inverted source behaviour in the sense that the strength of the reflection component is anti-correlated with the $2-10 \mathrm{keV}$ flux might be another hint for a scenario in which the reprocessing occurs at large distance to the central engine of the AGN, and therefore a correlation of the reflected component and the continuum would appear with a large delay (Malzac \& Petrucci 2002).
Based on the data analysed here, this distance appears to be 1.5 light years, as there is no variability observable in the 2-100 keV X-ray luminosity, but significant variation in the reflection component. This would place the material relevant for the reprocessing at a distance of $0.55 \mathrm{pc}$, at the outer regions of the accretion disc. This being said, we cannot exclude that the flux varied in between the observations presented here, therefore the distance has to be taken as an upper limit.

\subsection{Accretion power in MCG-05-23-016}

MCG-05-23-016 presents a rather unique case. Its spectrum is, at least to the first order, nearly constant in shape and luminosity, with a possibly variable reflection component $(R=0 \ldots 1)$ and high energy cut-off $\left(E_{\mathrm{C}}=50 \ldots 120 \mathrm{keV}\right)$. The over all luminosity is low. Using the X-ray luminosity of $L_{2-200 \mathrm{keV}}=$ $10^{44} \mathrm{erg} \mathrm{s}^{-1}$ as a proxy for the bolometric luminosity, the small black hole mass of $2 \times 10^{6} M_{\odot}$ (Whang \& Zhang 2007) leads to a large Eddington ratio of $L_{\text {bol }} / L_{\text {Edd }} \gtrsim 0.4$. As the UV/optical emission is at least of the same order as the X-ray one, it is more likely that the Eddington ratio is as high as $L_{\text {bol }} / L_{\text {Edd }} \gtrsim 0.8$. Considering the error on the mass determination, this translates in a range of $L_{\mathrm{bol}} / L_{\mathrm{Edd}}=0.2-5$.

An independent way to determine the mass of a black hole is to study its temporal behaviour. Following McHardy et al. (2006), the detection of a break in the X-ray power spectral density (PSD) of an AGN would allow to estimate the black hole mass thanks to the relation $T_{\mathrm{B}}=2.1 \log \left(M_{\mathrm{BH}} \times 10^{-6} M_{\odot}^{-1}\right)-$ $0.98 \log \left(L_{\text {bol }} \times 10^{-44} \mathrm{erg}^{-1} \mathrm{~s}\right)-2.32$. Using the data collected by RXTE/PCA and XMM-Newton/PN between 1996 and 2005, we estimated the X-ray PSD and structure function (RXTE data only) of MCG-05-23-016. The structure function has the advantage of working in the time domain and therefore being less sensitive to alias and windowing problems than the Fourier analysis. The PSD was calculated for each observation longer than $10 \mathrm{ks}$ (on $200 \mathrm{~s}$ binned light curves), and the PSDs obtained were averaged and binned in logarithmically spaced bins (e.g. Uttley et al. 2002). The final PSD shows a flattening between $2 \times 10^{-5}-10^{-4} \mathrm{~Hz}(10-50 \mathrm{ks})$, close to the minimum frequencies sampled by these data $\left(\sim 7 \times 10^{-6} \mathrm{~Hz}\right)$. Also the structure function presents the hint of a break, but rather at $\sim 3.5 \mathrm{ks}$. The lack of a longer-term monitoring prevents us from drawing a firm conclusion about the presence and the position of the break. Assuming a break in the range $0.04-0.6$ days $(3.5-50 \mathrm{ks})$, we achieve a black hole mass of $M_{\mathrm{BH}}=1.84-3.3 \times 10^{7} M_{\odot}$. It has to be taken into account though that for the given bolometric luminosity the formula does not allow for black hole masses lower than $1.8 \times 10^{7} M_{\odot}$, even when the break time approaches zero. This means that it implies an upper limit for the Eddington ratio of $L_{\text {bol }} / L_{\text {Edd }} \lesssim 0.1$ at a luminosity of $L_{\text {bol }}=2 \times 10^{44} \mathrm{erg} \mathrm{s}^{-1}$, which might be exceeded in the case presented here. It also means that the mass estimate of MCG-05-23-016 based on the temporal behaviour is at the lower limit of the possible values.

Figure 5 shows the SED of the source for the data presented here, corrected for Galactic absorption of $N_{\mathrm{H}, \mathrm{Gal}}=8 \times 10^{20} \mathrm{~cm}^{-2}$ in the line of sight, giving an extinction of $A_{V}=N_{\mathrm{H}} / 1.79 \times$ $10^{21} \mathrm{~cm}^{-2}=0.45 \mathrm{mag}$ (Predehl \& Schmitt 1995). The extinction in the UV is higher (e.g. $A_{\mathrm{U}}=0.74 \mathrm{mag}$ ), while the effect is insignificant in the near infrared with e.g. $A_{K}=0.05$ mag (Schlegel et al. 1998). To the simultaneous data we added previous observations in the $J, H$, and $K$ s band from the 2MASS and VLA observations at $6 \mathrm{~cm}$ and $20 \mathrm{~cm}$ (Ulvestad et al. 1984) for comparison. The SED indicates that the bolometric 


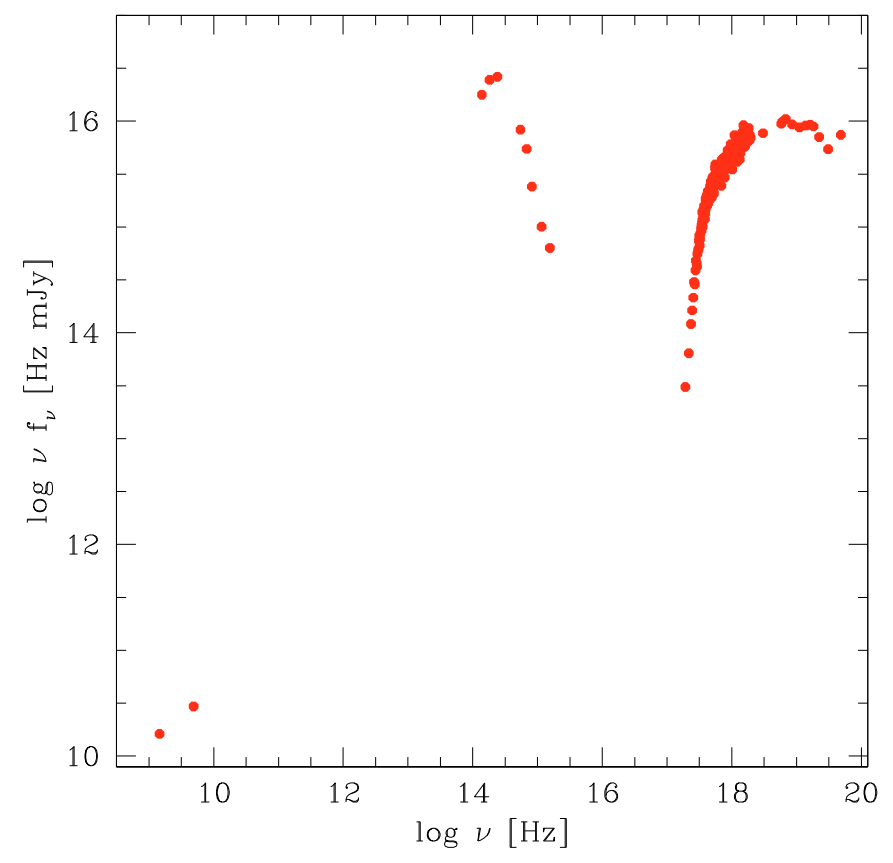

Fig. 5. Spectral energy distribution of the combined data set, including Swift/UVOT optical and UV data as well as Swift/XRT soft X-ray, and INTEGRAL JEM-X and IBIS/ISGRI hard X-ray data. In addition, 2MASS measurements $(J, H$, and $K \mathrm{~s})$ and radio data $(6 \mathrm{~cm}$ and $20 \mathrm{~cm})$ have been added. Data have been corrected for Galactic hydrogen column density, but not for intrinsic absorption.

luminosity is probably $L_{\text {bol }}>2 L_{X}$. Even considering the uncertainty of the mass determination, the source cannot be assumed to be a typical low-luminosity AGN, which exhibits Eddington ratios in the range $10^{-3}$ to $10^{-6}$ (Ho 1999), and the Seyfert 1.9 shows an Eddington ratio $10^{8}$ times larger than that of $\mathrm{Sgr} \mathrm{A}^{*}$ which has a similar mass of $M=3.3 \times 10^{6} M_{\odot}$ (Schödel et al. 2002). If we assume that the mass is in fact $M \lesssim 5 \times 10^{6} M_{\odot}$ then the Eddington ratio of $L_{\mathrm{bol}} / L_{\mathrm{Edd}} \gtrsim 0.8$ is also remarkable when compared to other Seyfert galaxies. Woo \& Urry (2002) list a total of 32 Seyfert galaxies with black hole masses lower than $10^{7} M_{\odot}$ and only 3 of them have $L_{\text {bol }} / L_{\text {Edd }} \gtrsim 1$, whereas the average of these lower mass black holes is $L_{\text {bol }} / L_{\text {Edd }}=0.30 \pm 0.05$. In the Galactic equivalent, the hard state is usually reached at small Eddington ratios, like $L_{\mathrm{bol}} / L_{\mathrm{Edd}} \simeq 10^{-3}$ in the case of XTE J1118+480 (Esin et al. 2001), and 0.003-0.2 for XTE J1550+564 (Done \& Gierliński 2003). In fact the SED of the X-ray nova XTE J1118+480 in the low state appears very similar to the one of MCG-05-23-016. In XTE J1118+480 the thermal disc component has a temperature as low as $24 \mathrm{eV}$. Considering that the temperature scales with $T \propto M^{-0.25}$, the disc emission of the Seyfert can be expected at much lower frequencies and can be hidden in the SED component peaking around $10^{14.5} \mathrm{~Hz}$. XTE J1118+480 also shows a power law component dominating the spectrum in the $0.4-160 \mathrm{keV}$ range with a photon index of $\Gamma=1.8$ (McClintock et al. 2001). The overall SED in this object can be explained by an advection dominated accrection flow (ADAF) model at 2\% Eddington ratio Esin et al. (2001). Although the SED of MCG-05-23-016 appears to resemble the one of XTE 1118+480, its high Eddington ratio is unlikely to be arising from a radiative inefficient accretion as described in the ADAF. An object with higher Eddington ratio also in the hard state is GX 339-4, which reaches $L_{\text {bol }} / L_{\text {Edd }}=0.25$ in the hard state in some cases before the state transition into the high-soft state (Zdziarski et al. 2004), consistent with MCG-05-23-016, although on average GX 339-4 reaches only an Eddington ratio of 0.015 and 0.05 during quiescence and during outburst, respectively. Considering a time scale for accretion rate changes in GX 339-4 of 1000 days (Zdziarski et al. 2004), this would correspond to time scales of $\sim 1 \mathrm{Myr}$ in the case of MCG-0523-016, as the variation times scale with the mass of the black hole.

Objects which are thought to exhibit extremely high Eddington ratios are the ultra-luminous X-ray sources (ULX). The true nature of these objects is still unclear though. Considering their luminosity, they are either intermediate mass black holes (IMBH) with a central mass as high as 100-100000 $M_{\odot}$ (e.g. Colbert \& Mushotzky 1999), or alternatively they are operating at very high Eddington ratio, as large as $L_{\text {bol }} / L_{\text {Edd }}>20$ (Soria et al. 2007; Roberts 2007). ULX also show hard spectra and low temperature disc similar to XTE J1550-564 in very high state where the disc temperature decreases (e.g. Kubota \& Done 2004). A study of Chandra data on ULX showed that the spectra can often be fitted by a simple power-law model, without evidence for thermal accretion disc components (Berghea et al. 2008), similar to MCG05-23-016. In some cases ULX show a disc component, e.g. the XMM-Newton observation of two ULX in NGC 1313 revealed soft components which are well fitted by multicolor disc blackbody models with color temperatures of $k T \simeq 150 \mathrm{eV}$.

An alternative model for the accretion process onto black holes has been proposed by Courvoisier \& Türler (2005), in which the accretion flows consist of different elements (clumps) which have velocities that may differ substantially. As a consequence, collisions between these clumps will appear when the clumps are close to the central object, resulting in radiation. In the case of MCG-05-23-016, this model results in lowenergetic collisions, which is also indicated by the missing variability in the $U V$ band as seen by Swift/UVOT, where these collisions should cause flux variations.

Low mass AGN like MCG-05-23-016 operating at high Eddington rate might be an early state in the evolution towards high-mass black holes as seen in quasars. As the highest measured redshift of a quasar to date is $z=6.43$ (Willott et al. 2007), we can assume that these objects with black hole masses of $M>10^{8} M_{\odot}$ appear in the Universe around $z \sim 7$. If we assume the formation of the first heavy black holes with $M \sim 10^{6} M_{\odot}$ at redshift $z=10$, we indeed need high mass accretion rates. An object like MCG-05-23-016, if we consider its mass to be indeed $M \lesssim 5 \times 10^{6} M_{\odot}$, with a constant Eddington ratio of $L_{\mathrm{bol}} / L_{\mathrm{Edd}}=0.8$ and starting black hole mass of $M(z=10)=$ $10^{6} M_{\odot}$ would reach a mass of $M(z=7)=4 \times 10^{8} M_{\odot}$. However, this would require not only the existence of a super massive black hole with $M \sim 10^{6} M_{\odot}$ at redshift $z=10$, but also a high accretion rate over a time span of $3 \times 10^{8} \mathrm{yrs}$. But even at a duty cycle of only $20 \%$ for AGN activity at $z \geq 7$, objects like MCG-05-23-016 can evolve to $10^{8} M_{\odot}$, and it has to be taken into account that the duty cycle of AGN is likely to be larger in the high-redshift Universe (Wang et al. 2008). On the other hand, it has to be considered that the environment in which the AGN grows at redshifts $z>7$ might be significantly different than the one we observe MCG -05-23-016 at in the local Universe.

\section{Conclusions}

The Seyfert 1.9 galaxy MCG-05-23-016 shows evidence for a variable reflection component, variable plasma temperature and high-energy cut-off energy. The combined data set presented here shows a low reflection component $(R<0.3)$ compared 
to previous studies which show a value as high as $R=1$. The data require a high-energy cut-off at $72 \mathrm{keV}$, but one observation shows an undisturbed power-law up to $E>100 \mathrm{keV}$. Tighter constraints on the hard X-ray spectrum and the effect of Compton reflection will be only achievable with focusing optics, as they will be provided by the future missions Simbol-X, NUSTAR, and NEXT.

During the observations the over all luminosity of the hard $\mathrm{X}$-ray emission remained rather constant, as did the UV/optical emission. The spectral energy distribution shows similarities with the one of the X-ray nova XTE J1118+480 in the low-state with the peaks of the SED shifted according to what one would expect from a scaling of $T \propto M^{-0.25}$. While XTE J1118+480 is assumed to accrete at low efficiency, perhaps through an advection dominated accretion flow, the Seyfert core is likely to be in a radiative efficient state. The low mass of the central black hole implies that the accretion onto the central engine takes place at a high Eddington ratio. An equivalent in the Galactic black hole class appears to be GX 339-4 in hard state before state transition, although the efficiency of the accretion in MCG-05-23016 is still higher. In this respect this source shows similarities to ULX. These high accretion flows are a key element in order to understand the rapid growth of black holes in the early Universe. Objects like MCG-05-23-016 might indicate the early stages of super massive black holes, in which a strong accretion flow feeds the central engine. With an Eddington ratio of $\gtrsim 0.8$, objects like the one presented here can develop into quasars with $10^{8} M_{\odot}$, even considering a duty cycle of only $20 \%$, within a time span of $3 \times 10^{8}$ yrs.

Acknowledgements. We thank the referee Chris Done who gave valuable advice which helped us to improve this paper. This research has made use of the NASA/IPAC Extragalactic Database (NED) which is operated by the Jet Propulsion Laboratory.

\section{References}

Arnaud, K. A. 1996, in Astronomical Data Analysis Software and Systems V, ed. G. Jacoby, \& J. Barnes, ASP Conf. Ser., 17, 101

Balestra, I., Bianchi, S., \& Matt, G. 2004, A\&A, 415, 437

Beckmann, V., Barthelmy, S. D., Courvoisier, T. J.-L., et al. 2007, A\&A, 475, 827

Berghea, C. T., Weaver, K. A., Colbert, E. J. M., \& Roberts, T. P. 2008, ApJ, 687, 471

Braito, V., Reeves, J. N., Dewangan, G. C., et al. 2007, ApJ, 670, 978
Burrows, D. N., Hill, J. E., Nousek, J. A., et al. 2005, Space Sci. Rev., 120, 165 Colbert, E. J. M., \& Mushotzky, R. F. 1999, ApJ, 519, 89

Courvoisier, T. J.-L., \& Türler, M. 2005, A\&A, 444, 417

Courvoisier, T. J.-L., Walter, R., Beckmann, V., et al. 2003, A\&A, 411, L53

Dadina, M. 2007, A\&A, 461, 1209

Done, C., \& Gierliński, M. 2003, MNRAS, 342, 1041

Esin, A. A., McClintock, J. E., Drake, J. J., et al. 2001, ApJ, 555, 483

Gebhardt, K., Bender, R., Bower, G., et al. 2000, ApJ, 539, L13

Gehrels, N., Chincarini, G., \& Giommi, P. 2004, ApJ, 611, 1005

Greene, J. E., \& Ho, L. C. 2005, ApJ, 627, 721

Ho, L. C. 1999, ApJ, 516, 672

Ibragimov, A., Poutanen, J., Gilfanov, M., Zdziarski, A. A., \& Shrader, C. R. 2005, MNRAS, 362, 1435

Kashlinsky, A., Arendt, R. G., Mather, J., \& Moseley, S. H. 2005, Nature, 438, 45

Kubota, A., \& Done, C. 2004, MNRAS, 353, 980

Lebrun, F., Leray, J. P., Lavocat, P., et al. 2003, A\&A, 411, L141

Lund, N., Budtz-Jorgensen, C., Westergaard, N. J., et al. 2003, A\&A, 411, L231

Magdziarz, P., \& Zdziarski, A. A. 1995, MNRAS, 273, 837

Malzac, J., \& Petrucci, P. O. 2002, MNRAS, 336, 1209

Mattson, B. J., \& Weaver, K. A. 2004, ApJ, 601, 771

McClintock, J. E., Haswell, C. A., Garcia, M. R., et al. 2001, ApJ, 555, 447

McHardy, I. M., Koerding, E., Knigge, C., Uttley, P., \& Fender, R. P. 2006, Nature, 444, 730

Miller, J. M., Fabbiano, G., Miller, M. C., \& Fabian, A. C. 2003, ApJ, 585, L37

Molina, M., Malizia, A., Bassani, L., et al. 2006, MNRAS, 371, 821

Predehl, P., \& Schmitt, J. H. M. M. 1995, A\&A, 293, 889

Poutanen, J., \& Svensson, R. 1996, ApJ, 470, 249

Reeves, J. N., Awaki, H., Dewangan, G. C., et al. 2007, PASJ, 59, 301

Roberts, T. P. 2007, Ap\&SS, 311, 203

Roming, P. W. A., Kennedy, T. E., Mason, K. O., et al. 2005, Space Sci. Rev., 120,95

Ross, R. R., \& Fabian, A. C. 2005, MNRAS, 358, 211

Schlegel, D. J., Finkbeiner, D. P., \& Davis, M. 1998, ApJ, 500, 525

Schödel, R., Ott, T., Genzel, R., et al. 2002, ApJ, 596, 1015

Soldi, S., Beckmann, V., Bassani, L., et al. 2005, A\&A, 444, 431

Soria, R., Baldi, A., Risaliti, G., et al. 2007, MNRAS, 379, 1313

Titarchuk, L. 1994, ApJ, 434, 570

Tremaine, S., Gebhardt, K., Bender, R., et al. 2002, ApJ, 574, 740

Tueller, J., Mushotzky, R. F., Barthelmy, S. D., et al. 2008, ApJ, 681, 113

Ulvestad, J. S., \& Wilson, A. S. 1984, ApJ, 285, 439

Uttley, P., \& McHardy, I. M. 2004, PThPS, 155, 170

Uttley, P., McHardy, I. M., \& Papadakis, I. E. 2002, MNRAS, 332, 231

Vedrenne, G., Roques, J.-P., Schönfelder, V., et al. 2003, A\&A, 411, L63

Wang, J.-M., \& Zhang, E. P. 2007, ApJ, 660, 1072

Wang, J.-M., Chen, Y.-M., Yan, C.-S., \& Hu, C. 2008, ApJ, 673, L9

Willott, C. J., Delorme, P., Omont, A., et al. 2007, AJ, 134, 2435

Winkler, C., Courvoisier, T. J.-L., Di Cocco, G., et al. 2003, A\&A, 411, L1

Woo, J.-H., \& Urry, C. M. 2002, ApJ, 579, 530

Zdziarski, A. A., Lubiński, P., \& Smith, D. A. 1999, MNRAS, 303, L11

Zdziarski, A. A., Gierliński, M., Mikołajewska, J., et al. 2004, MNRAS, 351, 791 